\title{
On Maximum Differential Graph Coloring
}

\author{
Yifan $\mathrm{Hu}^{1}$, Stephen Kobourov ${ }^{2}$, and Sankar Veeramoni ${ }^{2}$ \\ 1 AT\&T Labs Research, Florham Park, NJ, USA \\ yifanhu@research.att.com \\ 2 Computer Science Dept., University of Arizona, Tucson, AZ, USA \\ \{kobourov, sankar\}@cs.arizona.edu
}

\begin{abstract}
We study the maximum differential graph coloring problem, in which the goal is to find a vertex labeling for a given undirected graph that maximizes the label difference along the edges. This problem has its origin in map coloring, where not all countries are necessarily contiguous. We define the differential chromatic number and establish the equivalence of the maximum differential coloring problem to that of $k$-Hamiltonian path. As computing the maximum differential coloring is NP-Complete, we describe an exact backtracking algorithm and a spectral-based heuristic. We also discuss lower bounds and upper bounds for the differential chromatic number for several classes of graphs.
\end{abstract}

\section{Introduction}

The Four Color Theorem states that only four colors are needed to color any map so that no neighboring countries share the same color. This theorem assumes that each country forms a contiguous region in the map. However, if countries in the map are not all contiguous then the result no longer holds [6]. Instead, this necessitates the use of a unique color for each country to avoid ambiguity. As a result, the number of colors needed is equal to the number of countries.

Given a map, we define the country graph $G=\{V, E\}$ to be the undirected graph where countries are nodes and two countries are connected by an edge if they share a nontrivial boundary. We then consider the problem of assigning colors to nodes of $G$ so that the color distance between nodes that share an edge is maximized. Figure 1 gives an illustration of this map coloring problem.

As not all colors make suitable choices for country colors, a good color palette is often a gradation of certain "map-like colors". Furthermore, if the final result is to be printed in black and white, then the color space is strictly $1 \mathrm{D}$. We therefore make the assumption that the colors form a line in the color space, and model our map coloring problem as one of node labeling of the graph, where the available labels are from set $C$ of all permutations $\{1,2, \cdots|V|\}\}$. This brings us to the maximum differential graph coloring problem, in which we aim to find a vertex labeling for a given undirected graph that maximizes the label difference along the edges in the graph. 

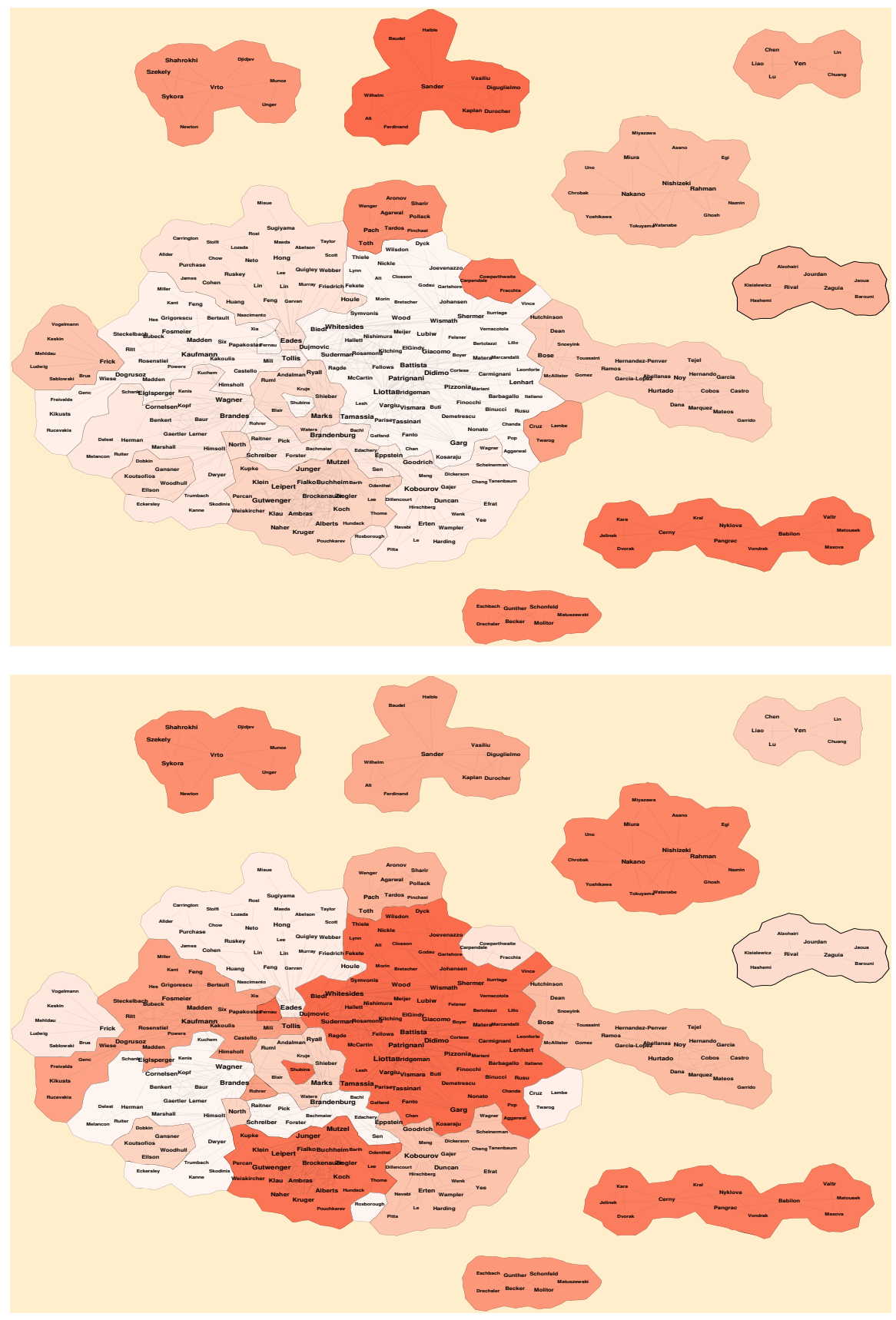

Fig. 1. Graph Drawing Symposia (1994-2004) co-authorship map with a single-hue color palette from ColorBrewer 2]: a random color assignment above and optimal below 
More formally, we are looking for a labeling function, a bijection $c: V \rightarrow$ $\{1,2, \ldots,|V|\}$, that solves the following MaxMin optimization problem:

$$
\max _{c \in C} \min _{\{i, j\} \in E} w_{i j}|c(i)-c(j)|
$$

Here $w_{i j}$ is a positive weight representing the importance of keeping the difference of the labels between nodes $i$ and $j$ large. To simplify the problem further, throughout this paper we assume $w_{i j}=1$.

Figure 2 illustrates the graph coloring problem. We use a color palette of yellow to blue, indexed from 1 to 9 . Figure 2(a) shows a map with 9 countries, with one non-contiguous country (its two components are at the center, and to the far right of the map). Figure2(b) shows the corresponding country graph. The node labeling is not optimal, with many adjacent nodes having label difference of 1 . Figure 2(c) gives the optimal node labeling, with a minimal label difference of 3 . The map in Figure 2(a) is in fact colored using this optimal coloring scheme, which gives distinctive colors for neighboring countries.

In this paper we define the differential chromatic number and show a correspondence between the maximum differential graph coloring problem and the Hamiltonian path problem in the complement graph. We also provide exact and heuristic algorithms for computing good solutions. In Section 3 we establish the relationship between this problem and that of finding a $k$-Hamiltonian path. Section 4 gives an exact algorithm and compares it with a heuristic algorithm on a number of well known graphs. Section 5 gives results for some special graphs. We conclude the paper with Section 6 .

\section{Related Work}

The problem of maximum differential coloring of graphs arises in the context of coloring a map in which not all regions are necessarily contiguous [7, 6. A variation of the differential graph coloring problem was studied by Dillencourt et al. 4, under the assumption that all colors in the color spectrum are available. This makes the problem continuous rather than discrete. A heuristic algorithm based on the force-directed model is used to select $|V|$ colors as far apart as possible in the 3-dimensional color space. However this algorithm cannot be used directly for the general map coloring problem, as maps are typically colored with "map-like colors", often light, pastel colors which come from a very restricted subset of the color spectrum. It may be possible to adapt the algorithm and apply it to a lower-dimensional color manifold, but because the algorithm is greedy it is more likely to converge to local minimum in lower dimensional space.

Finding a permutation that minimizes the labeling differences along the edges is well-studied problem in the context of minimum bandwidth or wavefront reduction ordering for sparse matrices. It is known that the bandwidth problem is NP-complete, with a reduction from 3SAT dating to 1975 [11. Moreover, it is NP-complete to find any constant approximation, even when restricting the problem to trees [1,15]. A number of effective heuristics for that problem have been proposed $8,9,13$. 


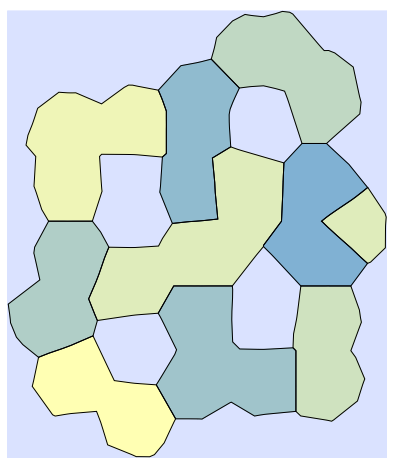

(a)

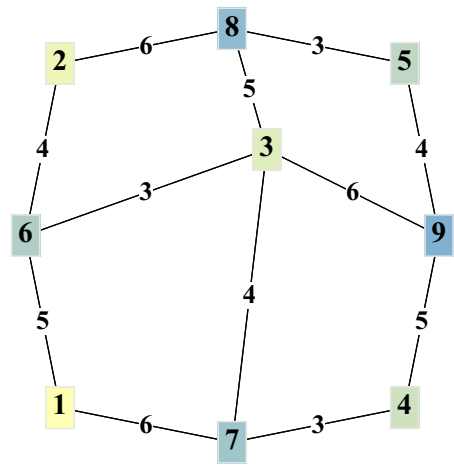

(c)

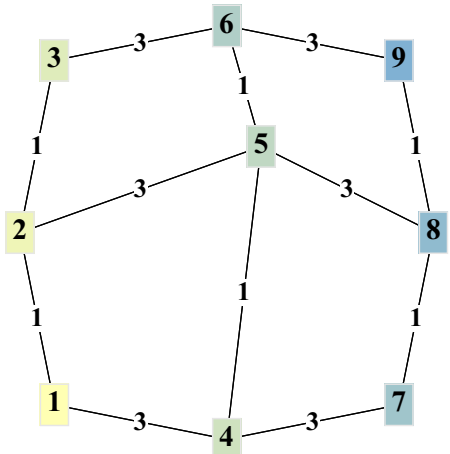

(b)

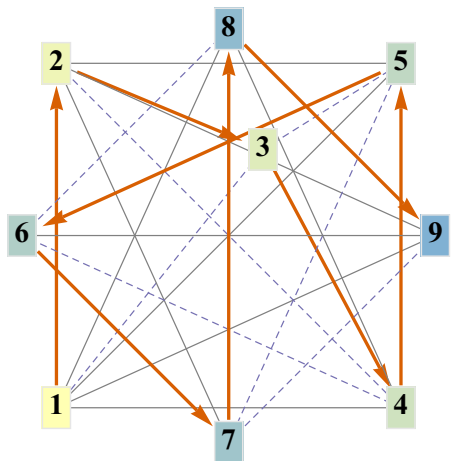

(d)

Fig. 2. An illustration of the graph coloring problem and its relation to a $k$-Hamiltonian path. A yellow to blue coloring scheme (indexed from 1 to 9 ) is used; nodes are labeled by color indices and edges are labeled with the absolute difference of of the adjacent nodes. (a) An input map. (b) The country graph $G$ corresponding to the input map; note that many adjacent nodes have very similar colors. (c) An optimal 3-differential coloring of the country graph. (d) The complement graph $\bar{G}$ corresponding to $G$ and a 2-Hamiltonian path. The Hamiltonian path $\{1,2, \ldots, 9\}$ is shown with orange arrows, and edges with labeling difference of 2 are shown as blue dashed lines. Note that the 2-Hamiltonian path of $\bar{G}$ is such that any two nodes of labeling difference $\leq 2$ form an edge of $\bar{G}$.

The complement of the bandwidth problem, that of maximizing the labeling difference along the edges, is the less well-known antibandwidth problem. Not surprisingly, it is also NP-Complete as shown in 1984 [10. Exact values for the antibandwidth are known for some Hamming graphs [5], as well as for meshes [14, hypercubes [12,16, and complete $k$-ary trees for odd values of $k[3]$.

There have been definitions for a graph being $k$-edge Hamiltonian and $k$ vertex Hamiltonian in the literature [17, which are defined as the graph having 
a Hamiltonian cycle after removing any $k$ edges or vertices, respectively. These concepts, though related, are different from our definition of $k$-Hamiltonian path, introduced in the next section.

\section{Maximum Differential Coloring and Hamiltonian Path}

Let the undirected graph of interest be $G=\{V, E\}$. We denote $\bar{G}$ as the complement of graph $G$, defined as $\bar{G}=\{\mathrm{V}, \bar{E}\}$, where $\bar{E}=\{\{i, j\} \mid i \neq j, i, j \in$ $V$, and $\{i, j\} \notin E\}$. In other words, $\bar{G}$ is the graph containing all nodes in $G$, and all edges that are not in $G$. We now formally define the maximum differential coloring problem.

Definition 1. A coloring of the nodes of $G$ is a bijection $c: V \rightarrow\{1,2, \ldots,|V|\}$. We denote the set of all colorings $C(G)$.

Definition 2. A $k$-differential coloring of $G$ is one in which the absolute coloring difference of the endpoints for any edge is $k$ or more. We denote the set of all such $k$-differential colorings $D C(G, k)=\{c|c \in C(G)| c,(i)-c(j) \mid \geq$ $k$ for all $\{i, j\} \in E\}$.

Definition 3. A graph is $k$-differential colorable if $D C(G, k) \neq \emptyset$.

Definition 4. If a graph is $k$-differential colorable, but not $(k+1)$-differential colorable, it has a differential chromatic number $k$, denoted as $d c(G)=k$.

Definition 5. A Hamiltonian path of $G$ is a bijection $p:\{1,2, \ldots,|V|\} \rightarrow V$, such that $\{p(i), p(i+1)\} \in E$ for all $i=1,2, \ldots,|V|-1$. We denote the set of all Hamiltonian paths $H(G)$.

The key insight in understanding the maximum differential coloring comes from observing a good differential coloring scheme. For example, in Figure 2(c), the minimum labeling difference between any two adjacency nodes is 3 . This means that any two nodes with labeling difference of $\leq 2$ can not form an edge in $G$, in other word they must form an edge in the complement of the graph, shown in Figure 2(d). Therefore the list of nodes induced by this coloring scheme of $G$, with labels $\{1,2, \ldots,|V|\}$, forms a Hamiltonian path in $\bar{G}$, shown in Figure 2(d) with orange arrows. Furthermore, this Hamiltonian path is such that any two nodes along the path with labeling difference of 2 are also connected by an edge of $\bar{G}$, shown in Figure 2(d) with dashed lines.

This observation leads to a natural extension of the concept of Hamiltonian path, which we call a $k$-Hamiltonian path.

Definition 6. A $k$-Hamiltonian path $(k \geq 1)$ of $G$ is a Hamiltonian path, such that each $i$-th node on the path is connected to the $j$-th node, if $|i-j| \leq k$. We define the set of $k$-Hamiltonian paths as $H(G, k)=\{p \mid p \in H(G)$, and $\{p(i), p(j)\} \in$ $E$ if $0 \leq i, j \leq|V|$ and $|i-j| \leq k\}$. Clearly $H(G)=H(G, 1)$. 
Based on our previous discussion, we can relate the $k$-differential coloring problem to that of finding a $(k-1)$-Hamiltonian path.

Theorem 1. A $k$-differential coloring $(k \geq 2)$ of a graph $G$ exists if and only if $a(k-1)$-Hamiltonian path exists in the complement of $G$, i.e., $D C(G, k) \neq \emptyset$ if and only if $H(\bar{G}, k-1) \neq \emptyset$. Furthermore, the inverse function of each element of $D C(G, k)$ is in $H(\bar{G}, k-1)$, and vice visa.

Proof. Suppose a $k$-differential coloring $c$ exists for $G$. Define the path, $p=c^{-1}$, so that it visits the vertices of the graph in order of their color index (i.e., the first vertex is that with color 1 , the second is that with color 2 and so on). Consider two nodes $u=p(i)$ and $v=p(j)$ such that $|i-j| \leq k-1$. Since the color difference of these two nodes in the original graph, $|c(u)-c(v)|=$ $|i-j| \leq k-1$, by the definition of $k$-differential coloring $(u, v)$ is not an edge of $G$, hence $\{u, v\}=\{p(i), p(j)\}$ is an edge of $\bar{G}$. It follows that $p=c^{-1}$ is a $(k-1)$-Hamiltonian path.

Conversely, suppose a $(k-1)$-Hamiltonian path $p$ exists for $\bar{G}$. Define a coloring $c=p^{-1}$, where the color index is given by the order in which a vertex appears in the path (i.e., color 1 is assigned to the first vertex along the path, color 2 to the second, and so on). Consider any edge $\{u, v\} \in E$. We prove that $|c(u)-c(v)| \geq k$. Assume that $|c(u)-c(v)|<k$. Let $i=c(u)$ and $j=c(v)$, then $|i-j|<k$, and $u=c^{-1}(i)=p(i)$ and $v=c^{-1}(j)=p(j)$. By the definition of a $(k-1)$-Hamiltonian path, $(u, v)$ must be an edge of $\bar{G}$, which is a contradiction with the fact that $\{u, v\} \in E$. It follows that $|c(u)-c(v)| \geq k$, and so $c=p^{-1}$ is a $k$-differential coloring of $G$.

This theorem immediately gives an upper bound for the differential chromatic number of a graph based on its maximum degree:

Corollary 1. A graph of maximum degree $\Delta(G)$ has a differential chromatic number of at most $|V|-\Delta(G)$.

Proof. $\bar{G}$ must have a node with degree $|V|-1-\Delta(G)$, therefore $H(\bar{G},|V|-$ $\Delta(G))=\emptyset$, or by Theorem $1 D C(G,|V|-\Delta(G)+1)=\emptyset$.

A special case of differential coloring is finding a scheme with maximum difference of 2 or more. Clearly:

Corollary 2. Finding a 2-differential coloring of a graph $G$ is equivalent to finding a Hamiltonian path of $\bar{G}$.

Given the equivalence between 2-differential coloring and Hamiltonian path, a number of well known results for Hamiltonian path can immediately be used to give results on 2-differential coloring.

Theorem 2. (Ore's Theorem) A graph with more than 2 nodes is Hamiltonian if, for each pair of non-adjacent nodes, the sum of their degrees is $|V|$ or greater.

Corollary 3. A graph with more than 2 nodes is 2-differential colorable if, for each pair of adjacent nodes, the sum of their degrees is $|V|-1$ or less. 
Theorem 3. (Dirac's Theorem). A graph with more than 2 nodes is Hamiltonian if each node has degree $|V| / 2$ or greater.

Corollary 4. A graph with more than 2 nodes is 2-differential colorable if each node has degree $|V| / 2-1$ or less.

Corollaries 3,4 confirm the intuition that a sparser graph has a better chance of being more differential colorable 11 The flip side of this intuition is that the complement graph needs to be denser. For a graph to be $k$-colorable, the complement graph must be well connected. The next theorem follows from a result in [12]:

Theorem 4. The complement of a $k$-differential colorable graph is $(k-1)$ connected.

Given the connection between finding a 2-differential coloring and a Hamiltonian path, it is not difficult to prove directly that the maximum differential coloring problem is NP-Complete. An equivalent result was established in 1984 in the context of the antibandwidth problem [10].

\section{Algorithms for Maximum Differential Coloring}

Theorem 1 provides a way to check whether a graph is $k$-differential colorable. The following kpath algorithm attempts to find a $k$-Hamiltonian path of $\bar{G}=$ $\{V, \bar{E}\}$ from a given node $i$. Before calling kpath the $k$-Hamiltonian path is initialized as $p=\emptyset$. Each call to kpath recursively tries to add a neighbor to the last node in the path, and checks that this maintains the $k$-path condition. If the condition is violated, the next neighbor is explored, or the algorithm backtracks. If a $k$-Hamiltonian path of $\bar{G}$ is found by the algorithm, we know that the graph $G$ is $(k+1)$-colorable.

As the exact algorithm requires exponential time, it is impractical for large graphs, where heuristic algorithms might be a better choice. Gansner et al. 6] proposed a heuristic based on a relaxation of the discrete MaxMin problem (1) into a continuous maximization problem of 2-norm:

$$
\max \sum_{\{i, j\} \in E} w_{i j}\left(c_{i}-c_{j}\right)^{2}, \text { subject to } \sum_{i \in V} c_{i}^{2}=1
$$

where $c \in R^{|V|}$. This continuous problem is solved when $c$ is the eigenvector corresponding to the largest eigenvalue of the weighted Laplacian of the graph. Once (2) is solved, the ordering defined by the eigenvector is used as an approximate solution for the MaxMin problem. This is followed by a greedy refinement algorithm which repeatedly swaps pairs of vertices, provided that the

\footnotetext{
${ }^{1}$ Even planar graphs, which have average degree less than 6 , may be tough to color well. Consider a star graph, where one vertex is connected to every other vertex in the graph. Regardless of the coloring, at least one pair of nodes will have a difference of at most one, thus showing that $d c(G)=1$.
} 


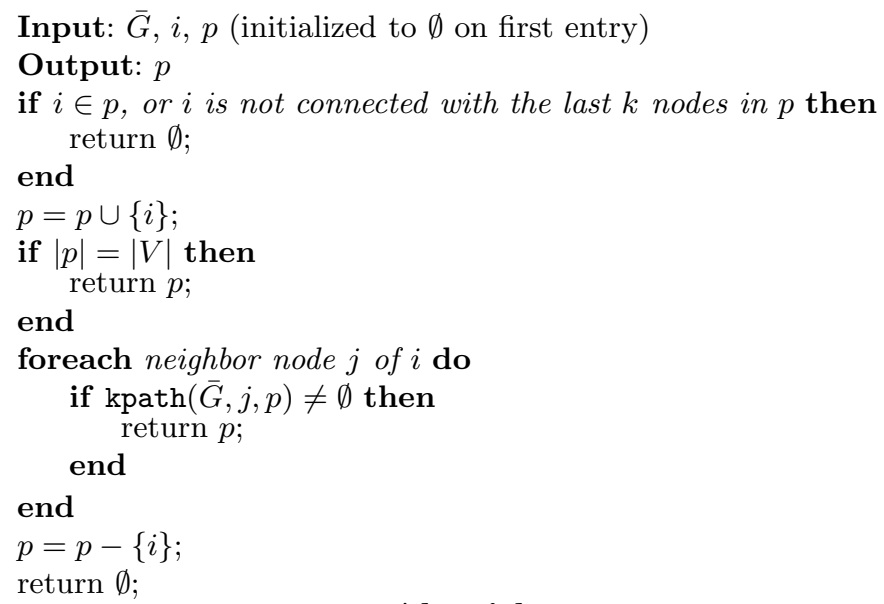

Algorithm 1. kpath

swap improves the coloring scheme. We call this algorithm GSpectral (Greedy Spectral). Table1 1 gives the chromatic number of some well-known graphs found by kpath, as well as an estimate of the differential chromatic number obtained by GSpectral. The exact algorithm kpath can be prohibitively expensive even for small graphs. For example, on the 60-node football graph (skeleton graph of a truncated icosahedron), one week of CPU time was not enough to find the exact differential chromatic number, whereas the greedy spectral algorithm gives a lower bound of 18 in a few milli-seconds. We note that the GSpectral algorithm often finds good solutions, though it rarely matches the optimal solution. We also tested GSpectral on larger grid graphs for which the differential chromatic number is known; see Section 5. For grid10 and grid20 graph, it gives an estimate of 30 and 124, and the actual differential chromatic numbers are 45 and 190, respectively. In both case the CPU time for GSpectral is less than 0.1 seconds.

\section{Differential Chromatic Numbers of Special Graphs}

Theorem 5. A line graph on $n$ nodes has differential chromatic number of $\lfloor n / 2\rfloor$. A cycle graph on $n$ nodes has differential chromatic number of $\lfloor(n-1) / 2\rfloor$.

Proof. Consider a line graph with even number of nodes labeled in order with $n / 2+1,1, n / 2+2,2, \ldots, n, n / 2$; see Fig. 3(top). This labeling is clearly a $n / 2$ differential coloring and it is easy to show that this is the best possible coloring. Take any labeling of this graph and consider the node labeled $n / 2$. Regardless of the labels of its neighbor(s) this node must induce an edge difference of at most $n / 2$ achieved if the neighbor(s) is labeled $n$. Hence the differential chromatic number can not be more than $n / 2$, that is, $d c(G)=n / 2=\lfloor n / 2\rfloor$. A similar argument also works when $n$ is odd; see Fig. 3 (bottom).

Now consider a cycle graph with an even number of vertices labeled in order with $1, n / 2+1,2, n / 2+2, \ldots, n / 2, n$; see Fig. 4(left). This labeling is clearly 
Table 1. Differential chromatic numbers given by kpath and by GSpectral

\begin{tabular}{|c|c|c|c|}
\hline name & drawing & $d c(G)$ & GSpectral \\
\hline petersen & & 3 & 3 \\
\hline hypercube & 14 & 4 & 4 \\
\hline binarytree3 & 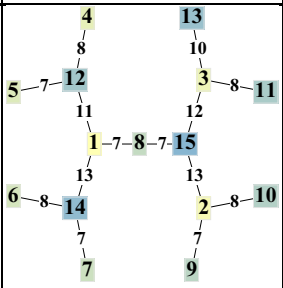 & 7 & 5 \\
\hline grid4 & 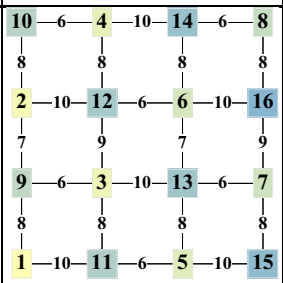 & 6 & 3 \\
\hline $\operatorname{grid} 5$ & 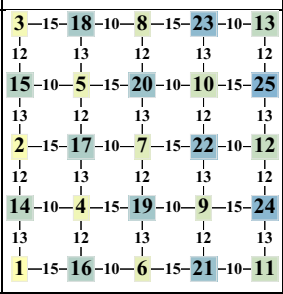 & 10 & 7 \\
\hline football & 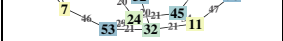 & - & 18 \\
\hline
\end{tabular}




$$
\begin{aligned}
& 6-5-1-6-7-5-2-6-8-5-3-6-9-5-4-6-10-5-5 \\
& 1-6-7-5-2-6-8-5-3-6-9-5-4-6-10-5-5-6-11-5-6
\end{aligned}
$$

Fig. 3. Optimal coloring of line graphs with 10 and 11 nodes

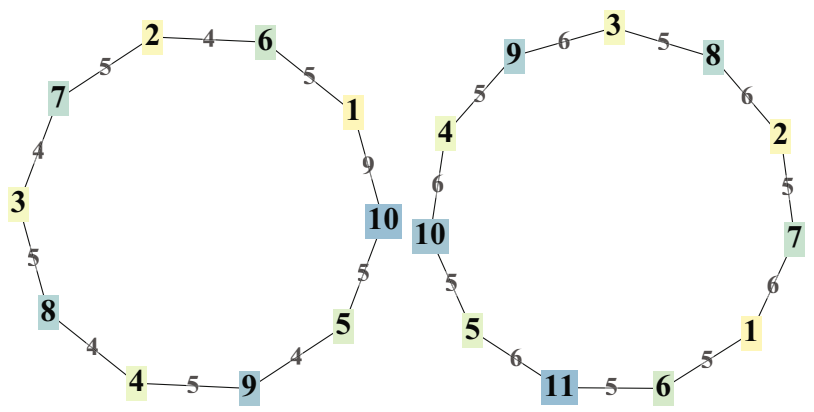

Fig. 4. Optimal coloring of ring graphs with 10 and 11 nodes

$n / 2-1=\lfloor(n-1) / 2\rfloor$ differential coloring and no better solution exists. Take any labeling of this graph and consider the node labeled $n / 2$. Regardless of the labels of its two neighbors this node must induce an edge difference of at most $n / 2-1$, achieved if the first neighbor is labeled $n$ and the second neighbor is 1 or $n-1$. A similar argument also works when $n$ is odd; see Fig. 4 (right).

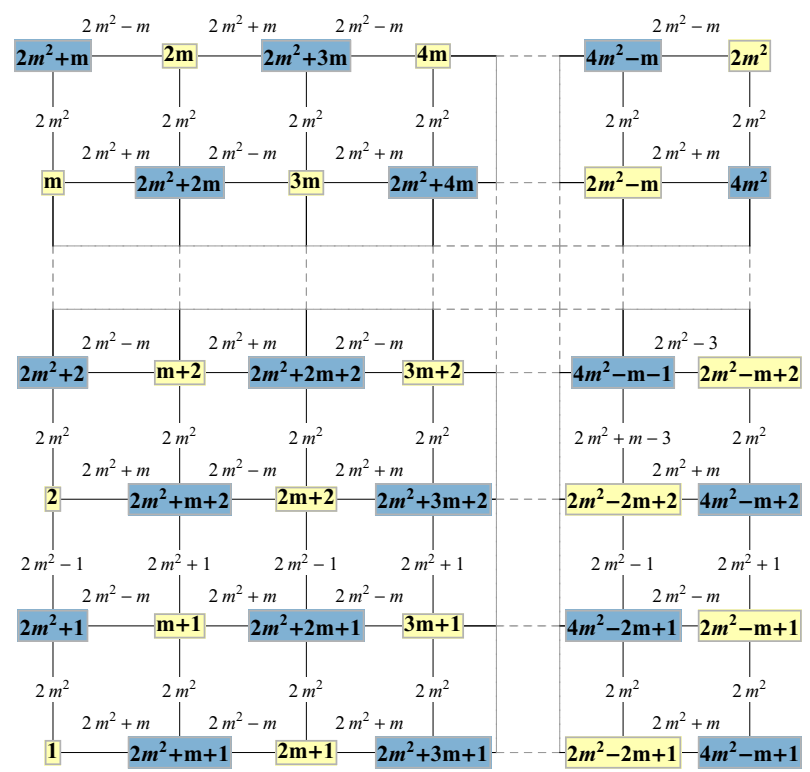

Fig. 5. Optimal coloring of $n \times n$-grids for even $n$ 

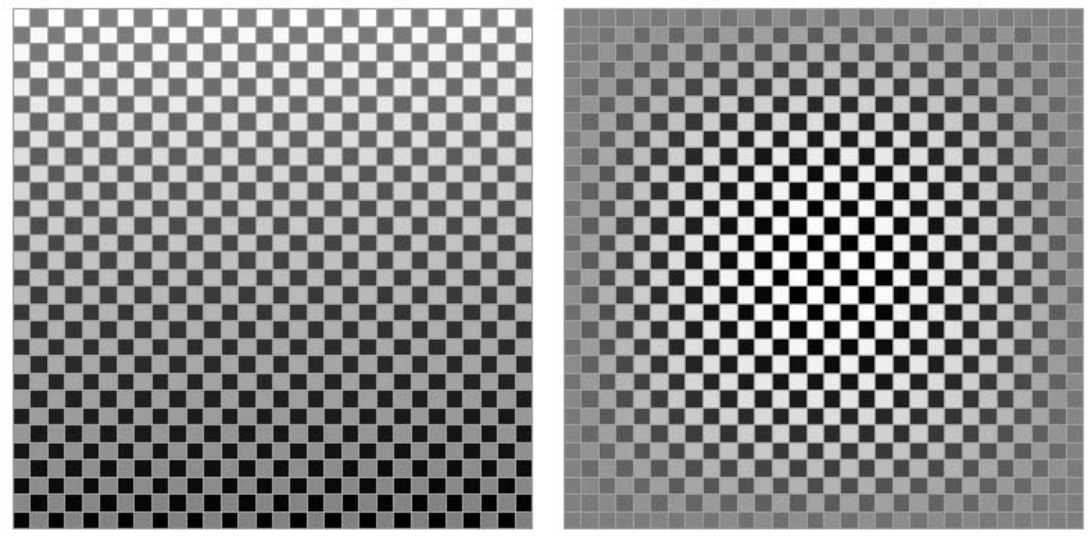

Fig. 6. Optimal grid map coloring (left) and one induced by the largest eigenvector (right)

Theorem 6. A grid graph of $n \times n$ nodes has differential chromatic number $\geq \frac{1}{2} n(n-1)$.

Proof. If $n$ is even, let $m=n / 2$, we can color the grid as shown in Fig. 5. Due to the symmetries in the numbering scheme, only five labeling differences are possible: $2 m^{2}-m, 2 m^{2}-1,2 m^{2}, 2 m^{2}+1,2 m^{2}+m$. The smallest is $2 m^{2}-m=\frac{1}{2} n(n-1)$. For odd $n$ there is also solution with only four label differences.

It turns out that $\frac{1}{2} n(n-1)$ is also an upper bound for the chromatic number of the $n \times n$ grid graph, making this result tight [12. It is informative to contrast an optimally colored grid map with a grid map that uses the coloring induced by the largest eigenvector; see Fig. 6. The eigenvector coloring provides good contrast between neighboring countries, particularly in the center. This indicates that GSpectral might ineed be a good practical heuristic for large graphs.

\section{Conclusion}

In this paper we introduced the maximum differential graph coloring problem which arises in the context of map coloring. We described exact and heuristic algorithms for this problem, and considered some special classes of graphs for which good solutions can be computed. We showed that this problem is related to that of finding a $k$-Hamiltonian path. There is also a close relationship between this problem and the antibandwidth problem.

We note that the results of this paper extend easily to the case when there are more than $|V|$ colors available: we can augment the graph with the same number of isolated "dummy" nodes as there are extra colors. We further note that countries that are not neighbors, but are nevertheless close (e.g., neighbor's 
neighbor), can also be forced to have distinctive colors by adding additional edges in the country graph linking these countries.

Throughout our paper we have been assuming that the edge weights in the country graph are uniform. That is, the importance of having a different color between any pair of adjacent countries in the map is the same. We would also like to investigate ways to handle non-uniform weights, where more importance can be placed on certain countries or certain types of adjacencies (e.g., long vs. short common borders).

\section{Acknowledgments}

We thank L'ubomír Török for pointing out the related work on the antibandwidth problem.

\section{References}

1. Blache, G., Karpinski, M., Wirtgen, J.: On approximation intractability of the bandwidth problem. Technical Report TR98-014, University of Bonn (1998)

2. Brewer, C.: ColorBrewer - Color Advice for Maps, http://www.colorbrewer .org

3. Calamoneri, T., Massini, A., Török, L., Vrt'o, I.: Antibandwidth of complete k-ary trees. Electronic Notes in Discrete Mathematics 24, 259-266 (2006)

4. Dillencourt, M.B., Eppstein, D., Goodrich, M.T.: Choosing colors for geometric graphs via color space embeddings. In: Kaufmann, M., Wagner, D. (eds.) GD 2006. LNCS, vol. 4372, pp. 294-305. Springer, Heidelberg (2007)

5. Dobrev, S., Královic, R., Pardubská, D., Török, L., Vrt'o, I.: Antibandwidth and cyclic antibandwidth of hamming graphs. Electronic Notes in Discrete Mathematics 34, 295-300 (2009)

6. Gansner, E.R., Hu, Y.F., Kobourov, S.G.: GMap: Visualizing graphs and clusters as maps. In: IEEE Pacific Visualization Symposium (PacVis), pp. 201-208 (2010)

7. Gansner, E.R., Hu, Y.F., Kobourov, S.G., Volinsky, C.: Putting recommendations on the map: visualizing clusters and relations. In: 3rd ACM Conference on Recommender Systems (RecSys), pp. 345-348 (2009)

8. Hu, Y.F., Scott, J.A.: A multilevel algorithm for wavefront reduction. SIAM Journal on Scientific Computing 23, 1352-1375 (2001)

9. Kumfert, G., Pothen, A.: Two improved algorithms for envelope and wavefront reduction. BIT 35, 1-32 (1997)

10. Leung, J., Vornberger, O., Witthoff, J.: On some variants of the bandwidth minimization problem. SIAM Journal on Computing 13, 650 (1984)

11. Papadimitriou, C.: The NP-Completeness of the bandwidth minimization problem. Computing 16, 263-270 (1975)

12. Raspaud, A., Schröder, H., Sýkora, O., Török, L., Vrt'o, I.: Antibandwidth and cyclic antibandwidth of meshes and hypercubes. Discrete Mathematics 309(11), 3541-3552 (2009)

13. Sloan, S.W.: An algorithm for profile and wavefront reduction of sparse matrices. International Journal for Numerical Methods in Engineering 23, 239-251 (1986)

14. Török, L., Vrt'o, I.: Antibandwidth of three-dimensional meshes. Discrete Mathematics 310(3), 505-510 (2010) 
15. Unger, W.: The complexity of the approximation of the bandwidth problem. In: Proceedings of the 39th Symposium on Foundations of Computer Science (FOCS), pp. 82-91 (1998)

16. Wang, X., Wu, X., Dumitrescu, S.: On explicit formulas for bandwidth and antibandwidth of hypercubes. Discrete Applied Mathematics 157(8), 1947-1952 (2009)

17. Wong, W.W., Wong, C.K.: Minimum K-Hamiltonian graphs. Journal of Graph Theory 8, 155-165 (2006) 